\title{
Oral Ingestion of Cannabis sativa: Risks, Benefits, and Effects on Malaria-Infected Hosts
}

Olugbenga Akinola, ${ }^{1-3}$ Elizabeth O. Ogbeche, ${ }^{1}$ Hidayah A. Olumoh-Abdul, ${ }^{4}$ Abdulmusawwir O. Alli-Oluwafuyi, ${ }^{1,2}$ Aboyeji L. Oyewole, ${ }^{2,5}$ Abdulbasit Amin, 2,5 Wahab Imam AbdulMajeed, 2,5 Olayemi Joseph Olajide, 2,6 Abdurrazaq B. Nafiu, ${ }^{2,5}$ Anoka A. Njan, ${ }^{1}$ Olufunke E. Olorundare, ${ }^{1}$ and Grace O. Gbotosho $3,7,8$

\begin{abstract}
Background: The emergence of a multidrug-resistant strain of Plasmodium falciparum (Pf Pailin) raises concern about malaria control strategies. Unfortunately, the role(s) of natural plants/remedies in curtailing malaria catastrophe remains uncertain. The claims of potential antimalarial activity of Cannabis sativa in vivo have not been well established nor the consequences defined. This study was, therefore, designed to evaluate the effects of whole cannabis consumption on malaria-infected host.

Methods: Thirty mice were inoculated with dose of $1 \times 10^{7}$ chloroquine-resistant Plasmodium berghei ANKAinfected erythrocyte and divided into six treatment groups. Cannabis diet formulations were prepared based on weighted percentages of dried cannabis and standard mice diet and the study animals were fed ad libitum. Chemosuppression of parasitemia, survival rates, parasite clearance, and recrudescence time were evaluated. Histopathological studies were performed on the prefrontal cortex (PFC) and hippocampus of the animals after 14 days' consumption of cannabis diet formulation by naive mice.

Results: There was a significant difference $(p<0.05)$ in the day-4 chemosuppression of parasitemia between the animals that were fed $C$. sativa and chloroquine relative to the untreated controls. There was also a significant difference in the survival rate ( $p<0.05)$ of animals fed C. sativa diet $(40 \%, 20 \%, 10 \%$, and $1 \%)$ in contrast to control animals on standard mice diet. A parasite clearance time of $2.18 \pm 0.4$ was recorded in the chloroquine treatment group, whereas recrudescence in chloroquine group occurred on day 7 . There were slight histomorphological changes in the PFC and cell densities of the dentate gyrus of the hippocampus of animals that were fed C. sativa. Conclusions: $C$. sativa displayed mild antimalarial activity in vivo. There was evident reduction in symptomatic manifestation of malaria disease, though unrelated to levels of parasitemia. This disease tolerance status may be beneficial, but may also constitute a transmission burden through asymptomatic carriage of parasites by habitual cannabis users.
\end{abstract}

Keywords: Cannabis therapeutics; malaria; disease tolerance; Plasmodium berghei; asymptomatic reservoirs

\section{Introduction}

Continuing efforts at combating and eliminating malaria have culminated in the dramatic reduction of the malaria burden, with an estimated 6.2 million malaria deaths averted between 2001 and 2015. ${ }^{1}$ The successes are, however, being threatened by a number of factors, including parasite drug resistance. ${ }^{2}$ Increasing reports of reduced parasite susceptibility and drug failures in

\footnotetext{
${ }^{1}$ Department of Pharmacology and Therapeutics, Faculty of Basic Medical Sciences, University of Ilorin, Ilorin, Nigeria.

${ }^{2}$ Biomedical Research Group, University of Ilorin, Ilorin, Nigeria.

${ }^{3}$ Malaria Research Laboratories, Institute for Advanced Medical Research and Training, College of Medicine, University of Ibadan, Ibadan, Nigeria.

${ }^{4}$ Department of Pharmacology and Toxicology, Faculty of Pharmacy, University of Ilorin, Ilorin, Nigeria.

Departments of ${ }^{5}$ Physiology and ${ }^{6}$ Anatomy, University of Ilorin, llorin, Nigeria.

${ }^{7}$ Department of Pharmacology and Therapeutics, University of Ibadan, Ibadan, Nigeria.

${ }^{8}$ Department of Pharmacology and Toxicology, Faculty of Pharmacy, University of Ibadan, Ibadan, Nigeria.
}

*Address correspondence to: Olugbenga Akinola, PhD, Department of Pharmacology and Therapeutics, Faculty of Basic Medical Sciences, University of Ilorin, Ilorin 24003, Nigeria, E-mail: akinola.o@unilorin.edu.ng; gbenga_akinola@ymail.com

\footnotetext{
(C) Olugbenga Akinola et al. 2018; Published by Mary Ann Liebert, Inc. This Open Access article is distributed under the terms of the Creative Commons License (http://creativecommons.org/licenses/by/4.0), which permits unrestricted use, distribution, and reproduction in any medium, provided the original work is properly cited.
} 
Southeast Asia due to parasite resistance to artemisininbased combination therapies ${ }^{3-9}$ now threaten malaria control efforts. The clinical consequences of artemisinin resistance may be dire, and more devastating in Africa, where $>90 \%$ of all malaria cases are recorded. ${ }^{10,11}$ More worrisome is the emergence of a multidrug-resistant strain of Plasmodium falciparum (Pf Pailin) from the Southeast Asia region now referred to as "Super Malaria."12

The alarming rate of drug failure necessitates the development and deployment of novel and highly efficacious antimalarial drugs. It is thus not surprising that numerous ongoing researches focus on finding newer chemotherapeutic agents that can combat and counter drug-resistant parasites. The role/use of natural plant remedies in curtailing the ever-burgeoning malaria catastrophe remains uncertain, as only a small fraction of plants have been evaluated and developed for their medicinal potentials. ${ }^{13}$ However, many communities in malaria-affected regions still rely on natural plants and herbs, despite documented concerns about efficacy and adverse effects. ${ }^{14}$

One plant of continuous controversy is Cannabis sativa, known mainly for its psychoactive effects but possesses other activities. ${ }^{15,16}$ Quite a number of medicinal preparations derived from $C$. sativa have been employed for a variety of ailments, including malaria, pain, fever, and rheumatism. ${ }^{17}$ The constituents of cannabis have also been linked to modulation of the immune system, ${ }^{18-22}$ and these immune responses play major roles in the pathophysiology of inflammatory diseases. ${ }^{23,24}$ The uncontrolled and irrational use of $C$. sativa by individuals may create a challenge for the management of diseases.

Cannabis, also known as marijuana, pot, weed, gbanaa, and dagga in different regions, is consumed in different ways, including smoking, vaporizing, and as tea and edibles. Onset of effects is within minutes when smoked and about 30-60 min when cooked and/or eaten. ${ }^{25}$ The prevalence of cannabis use by persons remains unstable due to limited and unreliable data from most countries. However, estimated figures show a high rate ( $>10 \%$ of population) of use in 19 out of 163 countries where data were available. ${ }^{26}$

Isolated constituents of cannabis, 4-acetoxycannabichromene, 5-acetyl-4-hydroxycannabigerol, and -1'S hydroxycannabinol, have been reported to possess mild-to-moderate antimalarial activity in vitro. ${ }^{27,28}$ Information on the potential of $C$. sativa in suppressing malaria infections in vivo is sparse, although users in malaria endemic regions claim that cannabis protects against malaria. ${ }^{29}$ The exploration of the potential antimalarial properties of $C$. sativa consumption in vivo is necessary to establish the favorable and/or unwanted effects of C. sativa in malaria-infected hosts. This information will be vital, as cannabinoids the major constituent of $C$. sativa plant are known to bind tightly to their receptors found in the brain. ${ }^{15,30}$ It is important to identify potentially valuable quantities of cannabis that may be useful against malaria infections. In this study, we attempt to model daily consumption of C. sativa by oral ingestion ad libitum, and its potential effects on chloroquine-resistant Plasmodium bergheiinfected mice.

\section{Materials and Methods}

Animals

Swiss albino mice of average weight of $24 \mathrm{~g}$ were utilized in all the experiments. The mice were used in accordance with the University of Ilorin "Guide for the care and use of laboratory animals."

\section{Plants}

Dried leaves, twigs, and seeds of the $C$. sativa plant were obtained from the National Drug Law Enforcement Agency (NDLEA), Kwara State Command, Nigeria. Identification, characterization, and validation of the plant were confirmed by evidence specialist at NDLEA, Kwara State Command.

\section{Preparation of cannabis-feed formulation}

The cannabis-standard feed formulations were prepared based on weighted percentages of dried C. sativa (leaves, twigs, and seeds in the ratio 6:3:1, respectively) and standard mice feed. This was presented as food to study mice ad libitum. A percentage weighed portion of milled C. sativa was mixed with corresponding percentage quantities of milled standard mice feed for different groups corresponding to $40 \%, 20 \%, 10 \%$, and $1 \%$ cannabis diet. The cannabis standard feed formulation was pelletized and labeled as "cannabis diet formulations" (C. sativa diet). The formulations were fed to study mice ad libitum.

\section{Determination of $50 \%$ lethal dose $\left(\mathrm{LD}_{50}\right)$}

A modification of Lorke's toxicity test ${ }^{31}$ was employed using 12 naive mice separated into three groups (I-III) and fed with $20 \%, 2 \%$, and $0.2 \%$ cannabis diet, respectively. The animals were closely monitored for signs of toxicity or lethality for $24 \mathrm{~h}$, after which the cannabis 
diet formulation was withdrawn and monitoring continued for 14 days. In the second phase, five animals were fed with $50 \%$ cannabis diet formulation ad libitum. These animals were also monitored for toxicity as already stated. In both phases, the animals fasted overnight before being fed the cannabis diet formulation.

\section{Antimalarial test in vivo}

A modified Peter's 4-day suppressive test $^{32}$ was employed in assessing antimalarial activity. Thirty experimental animals were intravenously inoculated with a dose of $1 \times 10^{7}$ chloroquine-resistant $P$. bergheiinfected erythrocyte. The animals were separated into six treatment groups of five mice per group comprising a positive control group (chloroquine $10 \mathrm{mg} / \mathrm{kg}$ ), a negative control group (water), and four groups based on percentages of cannabis in the diet formulation (40\%, $20 \%, 10 \%$, and $1 \%$ ) in respective groups (I-IV). Chloroquine $(10 \mathrm{mg} / \mathrm{kg})$ treatment commenced $24 \mathrm{~h}$ postinoculation and administered once daily for 3 days. Animals in the cannabis groups fasted overnight and were allowed access to the cannabis diet formulation ad libitum $24 \mathrm{~h}$ post-inoculation and throughout the duration of study. Animals in the chloroquine treatment and negative control group were fed standard mice diet.

Parasiticidal activity was assessed daily from day 1 post-infection till day 14, and then on days 17 and 21. Inhibition of parasite growth in chloroquinetreated and cannabis diet groups were calculated relative to parasitemia in the negative control. Parasite clearance and recrudescence times were also evaluated. Survival rates in all experimental groups were monitored daily and the mean survival time was calculated.

\section{Assessment of hematological parameters/indices}

Twenty naive mice were randomly divided into five groups of four animals each. The animals in groups I to IV were fed with cannabis diet formulation containing $40 \%, 20 \%, 10 \%$, and $1 \%$ cannabis for 14 days. Animals in group $\mathrm{V}$ were fed with standard mice feed. The animals were euthanized, blood collected through cardiac puncture into potassium-ethylenediamine tetraacetic acid bottles, and immediately analyzed for hematological parameters.

\section{Histology of brain tissues}

To avoid any potential effect of parasitemia or ambiguity of the action of $C$. sativa on brain tissues, uninfected naive mice were used for this experiment. Fifteen mice were randomly divided into five groups of three mice each. Animals in groups I to IV were fed with cannabis diet formulation containing $40 \%, 20 \%, 10 \%$ and $1 \%$ cannabis, respectively, for 14 days. Animals in group $\mathrm{V}$ were fed the standard mice diet. The animals were sacrificed by cervical dislocation, dissected frontally, and perfused with normal saline, to rid the tissues of blood. The whole brain was excised and rinsed in $30 \%$ sucrose solution, followed by fixation in $4 \%$ para formaldehyde in plain bottles placed on ice and later stored at $4^{\circ} \mathrm{C}$. After $24 \mathrm{~h}$, the prefrontal cortex (PFC) and hippocampus were excised and preserved in $30 \%$ sucrose solution at $4^{\circ} \mathrm{C}$ and later processed for histological staining with hematoxylin and eosin (H\&E) and $0.1 \%$ cresyl fast violet (CFV).

\section{Statistics}

Student $t$-test was used to analyze the differences in mean across all experiments. All experiments were conducted in triplicates and analyses were considered statistically significant at $p<0.05$.

\section{Results}

Lethal dose $\left(\mathrm{LD}_{50}\right)$

There were no signs of toxicity or lethality across all groups of mice fed with $50 \%, 20 \%, 2 \%$, and $0.2 \%$ cannabis diet formulation ad libitum within $24 \mathrm{~h}$. There was also no mortality recorded nor any observed sign of toxicity over a period of 2 weeks after the withdrawal of the cannabis diet even at the highest dose of $50 \%$ C. sativa in diet formulation.

\section{Assessment of intrinsic antimalarial activity}

The susceptibility profile of the parasites to cannabis, chloroquine treatment, and in the untreated negative controls is described in Figure 1. Parasitemia in the untreated control animals ranged from $17.01 \%$ on day 4 post-infection to a peak of $57.85 \%$ on day 8 when the last animal in the group died. There was no significant difference $(p>0.05)$ in the day- 4 suppression of parasitemia between the group of animals that were fed $1 \%$, $10 \%$, and $20 \%$ C. sativa diet formulation and the untreated controls (Fig. 1). However, there was a significant increase in day-4 parasite suppression in the group of animals that were fed with $40 \%$ C. sativa formulation $(p=0.001)$ relative to the control. There was a quantity-dependent effect in the day- 4 mean percentage chemosuppression across the groups with $31.68 \%$, $18.98 \%, 15.22 \%$, and $14.16 \%$ suppression of parasitemia in animals from groups I to IV, respectively. There was complete suppression of parasites on day 4 


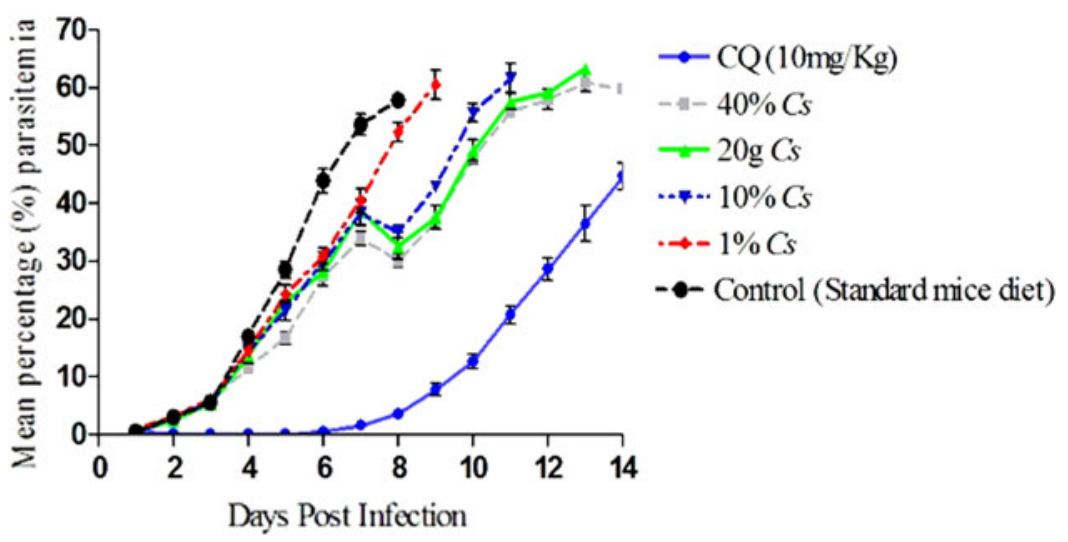

FIG. 1. Comparative response of treatment with chloroquine, oral ingestion of Cannabis sativa, and controls (standard mice diet) against chloroquine-resistant Plasmodium berghei ANKA strain parasites in mice.

in the chloroquine-treated group. Parasite clearance was recorded in the chloroquine treatment group with a mean parasite clearance time of $2.18 \pm 0.4$ days and recrudescence time of $6.80 \pm 0.6$ days. A reduction in parasitemia though not significant was observed on day 8 postinfection in the cannabis treatment groups of $40 \%, 20 \%, 10 \%$, and $1 \%$ cannabis diet.

\section{Survival rate of study animals}

The day 7 survival rates of animals fed on the cannabis diet formulation were consistent with the survival rates of chloroquine-treated animals (Table 1), despite the presence of significantly higher parasitemia in animals fed on cannabis diet formulation. There were significant differences in the day 14 survival rates between chloroquine treatment group and $C$. sativa treatment groups of $20 \%, 10 \%$, and $1 \%$ C. sativa diet. However, day 14 survival rates of animals fed with $40 \%$ C. sativa diet formulation were comparable with those with chloroquine-treated animals. The day 14 survival rate indicated a quantity-dependent effect of $C$. sativa con-

Table 1. Mean Survival Time and Percentage Survival of Malarial-Infected Animals Fed with Cannabis sativa

\begin{tabular}{lccc}
\hline & \multicolumn{2}{c}{$\begin{array}{c}\text { Percentage } \\
\text { survival (\%) }\end{array}$} & $\begin{array}{c}\text { Mean survival } \\
\text { time (days) }\end{array}$ \\
\hline Treatment group & Day 7 & Day 14 & \\
Chloroquine (10 mg/kg) & 100 & 100 & $17.2 \pm 1.8$ \\
$40 \%$ C. sativa & 100 & 60 & $15.2 \pm 2.3$ \\
$20 \%$ C. sativa & 100 & 20 & $12.8 \pm 1.4$ \\
$10 \%$ C. sativa & 100 & 0 & $11.2 \pm 1.8$ \\
$1 \%$ C. sativa & 100 & 0 & $9.4 \pm 1.3$ \\
Control (standard mice diet) & 60 & 0 & $7.6 \pm 1.1$ \\
\hline
\end{tabular}

sumption. There was a comparable mean survival time between the chloroquine treatment and the group of animals that consumed $40 \%$ cannabis diet. There was also a significant difference $(p<0.05)$ in mean survival time between all treatment groups and the untreated control (Table 1).

\section{Hematological indices}

There was no statistical significance $(p<0.05)$ in the hematological indices across all groups fed with $40 \%$, $20 \%, 10 \%$, and $1 \%$ cannabis diet formulations compared with the untreated controls fed standard mice diet. There was a remarkable decrease in platelet aggregation in the group of animals that were fed $40 \%$ C. sativa diet compared with other groups and controls, although this decrease was not significant. Table 2 gives the mean values of hematological parameters assayed in the different groups.

\section{Histology of the PFC}

Histological features of the animals in the control group did not show any altered morphology in panoramic presentation of the PFC layers. The cellular density within the control group appeared normal across all the layers with appreciable cellular projections. Animals fed $40 \%$ and $20 \%$ cannabis diet formulation showed slight changes in a quantity-dependent manner. These changes can be described by fragmented cells with observable pyknotic cells (yellow arrows), which is also associated with pronounced reduction of neuronal density in the $40 \%$ cannabis diet group. Animals in these groups $(40 \%$ and $20 \%$ C. sativa $)$ 
Table 2. Hematological Parameters Assessed in Animals Fed with Varying Quantities of Cannabis sativa Diet Formulation ad libitum for 14 Days and Controls Fed Standard Mice Diet

\begin{tabular}{|c|c|c|c|c|c|}
\hline \multirow[b]{2}{*}{ Parameter } & \multicolumn{5}{|c|}{ Mean \pm SEM } \\
\hline & $40 \%$ C. sativa & $20 \%$ C. sativa & $10 \%$ C. sativa & $1 \%$ C. sativa & Controls \\
\hline WBC & $3.12 \mathrm{e}^{3} \pm 1.1 \mathrm{e}^{3}$ & $3.0 e^{3} \pm 1.3 e^{3}$ & $6.7 \mathrm{e}^{3} \pm 1.6 \mathrm{e}^{3}$ & $3.4 \mathrm{e}^{3} \pm 7.5 \mathrm{e}^{3}$ & $3.5 \mathrm{e}^{3} \pm 4.2 \mathrm{e}^{3}$ \\
\hline $\mathrm{RBC}$ & $7.06 e^{6} \pm 7.7 e^{5}$ & $5.66 \mathrm{e}^{6} \pm 1.1 \mathrm{e}^{5}$ & $7.47 e^{6} \pm 1.6 e^{5}$ & $5.54 \mathrm{e}^{6} \pm 7.3 \mathrm{e}^{5}$ & $7.12 e^{6} \pm 9.2 e^{5}$ \\
\hline PLT & $6.23 e^{4} \pm 8.9 e^{4}$ & $5.72 e^{5} \pm 1.3 e^{5}$ & $6.43 e^{5} \pm 2.8 e^{5}$ & $6.39 e^{5} \pm 1.6 e^{5}$ & $7.16 e^{5} \pm 2.5 e^{5}$ \\
\hline LYM & $2.8 e^{3} \pm 9.5 e^{2}$ & $4.45 e^{3} \pm 1.8 e^{3}$ & $5.75 \mathrm{e}^{3} \pm 1.3 \mathrm{e}^{3}$ & $2.78 \mathrm{e}^{3} \pm 5.4 \mathrm{e}^{2}$ & $3.1 \mathrm{e}^{3} \pm 3.2 \mathrm{e}^{2}$ \\
\hline $\mathrm{HCT}$ & $30.63 \pm 1.16$ & $28.13 \pm 6.27$ & $35.58 \pm 1.45$ & $34.60 \pm 2.90$ & $2.90 \pm 4.25$ \\
\hline MCV & $48.50 \pm 1.12$ & $47.90 \pm 0.95$ & $47.57 \pm 0.91$ & $47.43 \pm 1.01$ & $47.93 \pm 0.72$ \\
\hline $\mathrm{MCH}$ & $13.20 \pm 0.12$ & $12.97 \pm 0.56$ & $13.40 \pm 0.27$ & $12.97 \pm 0.32$ & $12.87 \pm 0.38$ \\
\hline $\mathrm{MCHC}$ & $27.20 \pm 0.69$ & $26.93 \pm 0.67$ & $28.20 \pm 0.47$ & $27.33 \pm 0.69$ & $26.83 \pm 0.44$ \\
\hline RDW & $30.03 \pm 1.66$ & $28.00 \pm 0.47$ & $29.43 \pm 0.09$ & $28.47 \pm 0.68$ & $30.07 \pm 0.37$ \\
\hline PDW & $7.83 \pm 0.09$ & $8.73 \pm 0.23$ & $7.13 \pm 0.20$ & $8.53 \pm 0.52$ & $8.02 \pm 0.26$ \\
\hline MPV & $6.40 \pm 0.06$ & $6.57 \pm 0.09$ & $5.90 \pm 0.10$ & $6.60 \pm 0.25$ & $4.85 \pm 0.18$ \\
\hline PLCR & $5.30 \pm 0.12$ & $5.37 \pm 0.52$ & $3.67 \pm 0.50$ & $5.33 \pm 0.89$ & $4.62 \pm 0.55$ \\
\hline $\mathrm{HGB}$ & $7.30 \pm 0.95$ & $7.40 \pm 1.63$ & $10.03 \pm 0.38$ & $9.23 \pm 1.27$ & $9.13 \pm 1.30$ \\
\hline
\end{tabular}

$\mathrm{HCT}$, total hematocrit; $\mathrm{HGB}$, hemoglobin levels; LYM, lymphocyte count; $\mathrm{MCH}$, mean corpuscular hemoglobin; MCHC, mean corpuscular hemoglobin concentration; MCV, mean corpuscular volume; MPV, mean platelet volume; PDW, platelet distribution width; PLCR, platelet large cell ratio; PLT, platelet count; RBC, red blood cell count; RDW, red cell distribution width; SEM, standard error of mean; WBC, white blood cell count.

also presented with chromatolytic neurons (red circle) with reduced/irregular Nissl body distribution that suggests proteolysis. Groups of animals fed with $10 \%$ and $1 \%$ C. sativa showed characteristically normal cellular density and typical histoarchitectural assortment. Figure 2 depicts the photomicrographs showing the general cytoarchitecture of the PFC of animals fed
$40 \%, 20 \%, 10 \%$, and $1 \%$ C. sativa diet for 14 days with $\mathrm{H} \& \mathrm{E}$ staining and CFV stains.

\section{Histology of the hippocampus}

The control group did not show any alteration in morphological presentation of the hippocampal layers across the various exposures and magnification
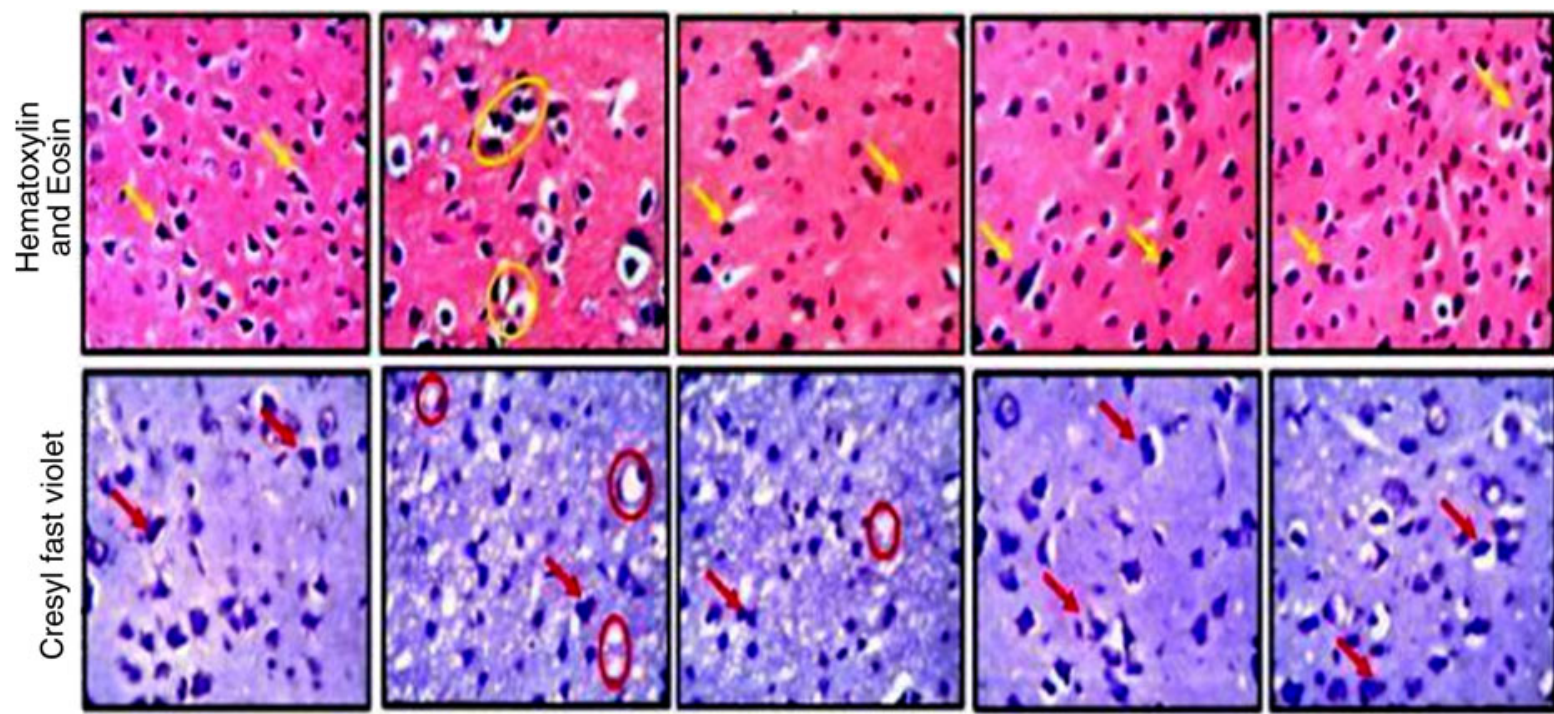

FIG. 2. Representative photomicrographs of the prefrontal cortex of animals fed cannabis diet and untreated controls. A high power magnification showing the general histomorphology (H\&E) and Nissl body characteristics (CFV) of animals fed $40 \%, 20 \%, 10 \%$, and $1 \%$ for 14 days. The pyknotic cells are identified by the yellow arrows, while the chromatolytic neurons are captured in red circles. The chromatolytic neurons were also captured in yellow circles but under H\&E staining, while the red arrows point to the pyknotic cell under the CFV staining. CFV, cresyl fast violet; H\&E, hematoxylin and eosin. 


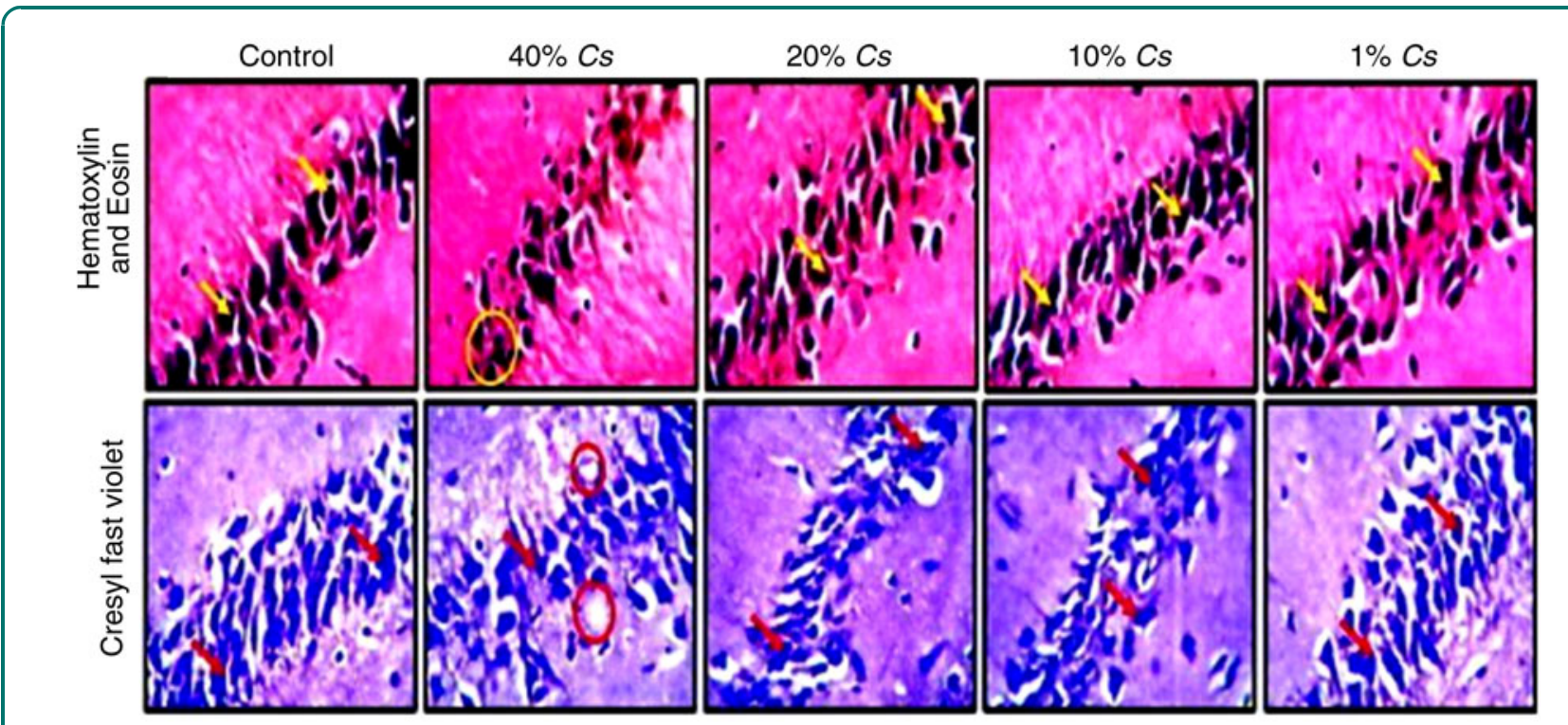

FIG. 3. Representative photomicrographs of CA region of the hippocampus of animals fed cannabis diet and untreated controls. A high power magnification showing the general the histomorphology (H\&E) and Nissl body characteristics (CFV) stains of animals fed $40 \%, 20 \%, 10 \%$, and $1 \%$ Cannabis sativa for 14 days. The large pyramidal cells are identified by yellow arrows, while the pyknotic cells can be spotted in yellow circles. The red arrows point at the large pyramidal cell, while the red circles show the pyknotic cells, both under CFV staining. CA, cornu ammonus.

$(\times 400)$. Groups of animals fed with $20 \%, 10 \%$, and $1 \%$ C. sativa showed normal architecture, characterized by large pyramidal cells (yellow arrows) with appreciable axonal projections across the intact neuropil. The histomorphology of the hippocampus in animals fed $40 \%$ C. sativa diet displayed moderate-to-mild chromatolytic changes characterized by clusters of pyknotic cells (yellow circles) and reduced neuronal density relative to the control group. Figure 3 shows the pictorial representation of the hippocampus stained with $\mathrm{H} \& \mathrm{E}$ and Nissl profile demonstration by CFV stain $(\times 400)$ across hippocampal sections of the brain in the group of animals labeled control (standard diet), 40\%, 20\%, $10 \%$, and $1 \%$ C. sativa diet formulation for 14 days.

\section{Discussion}

C. sativa is considered the most widely consumed illicit substance, ${ }^{26}$ largely so because of its usefulness in ameliorating a wide variety of ailments. ${ }^{17}$ The findings of this study outline the beneficial/risk interactions of whole $C$. sativa ingested orally by malaria-infected hosts. In this study, confiscated dried C. sativa was milled and mixed with standard mice feed and presented as food to mice ad libitum, to model the effects of oral cannabis consumption in habitual users. Dried leaves, twigs, and seeds were blended to mimic the preparations of cannabis in many places in Nigeria, where users often claim its protective potentials against infectious diseases such as malaria, alongside its psychoactive effects. In our survey of herbs in the treatment of malaria, cannabis is neither considered nor listed as part of ethnobotanical treatment of malaria in any part of Nigeria (unpublished).

Oral ingestion of $C$. sativa is the second most common method of $C$. sativa consumption, probably due to lack of toxic manifestations. This was demonstrated in this study with animals consuming blends of whole C. sativa up to $50 \%$ in the feed formulation for $24 \mathrm{~h}$ without any observable toxic concerns. This is corroborated by previous findings ${ }^{33}$ that assessed the tolerability of oral acute dose extracts of C. sativa, tetrahydrocannabinoids and cannabinoids, in humans. Also, cannabis is largely considered nontoxic, as no deaths have ever been reported due to cannabis overdose or unintended overconsumption, though it may possess risk for individuals with long-term use. ${ }^{25}$

C. sativa demonstrated poor in vivo chemosuppressive activity across all groups with mild-to-moderate antiparasitic activity in study animals. The phytocannabinoid metabolites of C. sativa may have contributed 
to the moderate antimalarial activity, as these compounds have been shown to possess antiplasmodial action in vitro. ${ }^{27,28}$ However, there might be other constituents of C. sativa that may have contributed to ${ }^{34}$ or reduced its activities by metabolic influences in vivo, as C. sativa is reported to mediate negative or positive characteristics on different pathological states. ${ }^{30,35,36}$ C. sativa exhibited a delayed suppressive effect on parasites in a quantity-dependent manner, as shown in our data from day 4 onward. The possibilities of bioaccumulated C. sativa constituents and/or its metabolites contributing to an improved parasite suppression were ruled out, as the animals were allowed continuous access to C. sativa, but there was no significant reduction in parasitemia until death.

C. sativa ingestion significantly increased the survival of infected animals in a quantity-dependent manner. Interestingly, the animals were not devoid of the high parasitic burden associated with malaria pathogenesis in mice. Although the antiparasitic action of C. sativa may not be comparable with that of chloroquine, the similarities in mean survival time in the C. sativa group fed $40 \%$ C. sativa may suggest the potential of cannabis in reducing pathogenicity of $P$. berghei. The disease tolerance status may not be unrelated to the immunomodulatory (pro- and anti-inflammatory) properties of cannabis, ${ }^{19,22,37,38}$ as have been shown in a study on cerebral malaria. ${ }^{39}$ The various properties from many different constituents of cannabis may have effected a trade-off of natural immune responses ${ }^{40}$ in the animals during malaria infections. This assertion is supported by previous reports that life-threatening manifestations of malaria are often as a result of dysregulation of host immune mechanisms. ${ }^{41,42}$ Irrespective of the potentials of ingested C. sativa to reduce the disease burden, infected animals eventually succumbed to malaria-related death. This shows that $C$. sativa is not curative against malaria infections, but may possess activity to enhance disease tolerance. Although death may also have been aided by the pathophysiological effects of $C$. sativa at quantities consumed by the study animals, the mechanism(s) involved remain(s) to be addressed.

Malaria parasites are known to preferentially invade and depend on the erythrocytes of the host for survival and proliferation. The influence of cannabis consumption on the blood cell integrity is necessary to assess the parasite infectivity profile in hosts. There were, however, no hematological changes in naive mice fed with cannabis to suggest any mecha- nism modulating invasion of red cells that may affect parasitemia.

The potential of cannabidiol to prevent behavioral changes in mice infected with $P$. berghei ANKA has been reported. ${ }^{39}$ In this study, the histomorphology of the PFC and hippocampus of naive animals showed evidence of morphological changes in a quantitydependent manner as previously suggested. ${ }^{43}$ These slight changes may be manifestations of the deleterious effects of $C$. sativa consumptions on the brain tissues, ${ }^{30}$ although it remains inconclusive as other adjuncts on the C. sativa plant may also be responsible. Although not assessed in this study, manifestations of PFC and the Hippocampal degeneration, such as, impaired motor function, short-term memory, paranoia, and psychosis may be apparent as previously reported. ${ }^{15}$ However, these degenerative effects may not play a role in the pathogenesis of cerebral malaria in cannabis users as the pathways and processes differ.

Observations from this study show the potential benefit of cannabis to mediate a disease tolerance effect during malarial infection, as demonstrated by the reduced negative effect of parasitemia on the animals. However, the host may become an active reservoir of the malaria parasite, capable of onward transmission of parasites within the population. This may pose a setback to malaria elimination, as it may contribute to potential resistance and cross-resistance from suboptimal concentrations of the mildly active antiplasmodial properties of $C$. sativa. The host may also become an "asymptomatic pool," harboring parasites that may become more virulent upon transmission to noncannabis users, as pathogens from asymptomatic carrier have been reported to be more virulent on symptomatic hosts. ${ }^{44,45}$

Although our study assessed the use of dried whole cannabis plant to model a common form of ingestion, the use of cannabis inflorescence may have produced a better antimalarial activity. Also, the ingestion route may have occluded the release of some other potential antimalarial constituents of cannabis, such as in its essential oils that contain high levels of terpinoids. Terpineol isolated from the oil of Tetradenia riparia plant has previously been reported to possess antimalarial activities in vitro. ${ }^{46}$ The variability in the chemical constituents of cannabis between cultivars may also produce slightly different results. Although there are several reports on the therapeutic use of cannabis constituents that can be validated, however, the consumption of whole cannabis is more prevalent globally. 


\section{Conclusion}

The potential of cannabis to reduce pathogenic manifestation of malaria disease in animals was established. However, there is the possibility of potential deleterious effects against malaria control efforts, thus, the continuous ingestion of cannabis blends may constitute more risk than benefits. Further research is needed to identify and isolate the different constituents of cannabis that may have mediated the benefits described in this study. Such findings may offer potential arrays into the modulation of vaccine activity and therapeutics that may limit the pathogenesis of severe and cerebral malaria incidences. With the new guidelines from the American Herbal Pharmacopeia on identification and standardization of cannabis for research, our understanding of this controversial plant will become clearer.

\section{Acknowledgments}

The authors thank the National Drug Law Enforcement Agency (NDLEA), Headquarter and Kwara State Command, Nigeria, for provision and validation of dried Cannabis sativa plant. The authors specially thank Mrs. S. Braimoh, Mrs. F.O. Oni, and Mr. A.O. Adesua for technical assistance.

\section{Ethical Approval}

This study was conducted at the Pharmacology and Therapeutics laboratory, University of Ilorin, Ilorin. Ethical approval was obtained from the University of Ilorin Ethical Review Committee (UERC/ASN/2017/ 857).

\section{Declaration on Data Set}

We declare that the data supporting the conclusions in this work are submitted with this article. Primary/raw data set will be made available on request.

\section{Author Disclosure Statement}

No competing financial interests exist.

\section{References}

1. World Health Organization. World Malaria Report 2016. Available at https://www.who.int/malaria/world_malaria_report_2016/en/ Accessed November 8, 2017.

2. World Health Organization. World Malaria Report 2017. Available at: https://www.who.int/malaria/world_malaria_report_2017/en/ Accessed January 21, 2018.

3. Dondorp AM, Nosten F, Yi P, et al. Artemisinin resistance in Plasmodium falciparum malaria. N Engl J Med. 2009;361:455-467.

4. Phyo AP, Nkhoma S, Stepniewska K, et al. Emergence of artemisininresistant malaria on the western border of Thailand: a longitudinal study. Lancet. 2012;379:1960-1966.

5. Bosman P, Stassijns J, Nackers F, et al. Plasmodium prevalence and artemisinin-resistant falciparum malaria in Preah Vihear Province,
Cambodia: a cross-sectional population-based study. Malar J. 2014; 13:394.

6. Gbotosho GO, Akinola O. A localised threat to an excellent antimalarial drug. Lancet Infect Dis. 2015;15:692-702.

7. Plowe CV, Ringwald P. Artemisinin resistance in Myanmar. Lancet Infect Dis. 2015;15:1001-1002.

8. Paloque L, Ramadani AP, Mercereau-Puijalon O, et al. Plasmodium falciparum: multifaceted resistance to artemisinins. Malar J. 2016;15:149.

9. Imwong M, Suwannasin K, Kunasol C, et al. The spread of artemisinin-resistant Plasmodium falciparum in the Greater Mekong subregion: a molecular epidemiology observational study. Lancet Infect Dis. 2017;17:491-497.

10. Woodrow CJ, White NJ. The clinical impact of artemisinin resistance in Southeast Asia and the potential for future spread. FEMS Microbiol Rev. 2017;41:34-48.

11. Alonso $P$, Noor $A M$. The global fight against malaria is at crossroads. Lancet. 2017;390:2532-2534.

12. Imwong M, Hien T, Thuy-Nhien NT, et al. Spread of a single multidrug resistant malaria parasite lineage (Pf Pailin) to Vietnam. Lancet Infect Dis. 2017;17:1022-1023.

13. Yuan $H, M a Q, Y e ~ L$, et al. The traditional medicine and modern medicine from natural products. Molecules. 2016;21:559.

14. Mojab F. Antimalarial natural products: a review. Avicenna J Phytomed. 2012;2:52-62.

15. Volkow ND, Baler RD, Compton WM and Weiss SRB. Adverse health effects of marijuana use. N Engl J Med. 2014;370:2219-2227.

16. Russo EB. Current therapeutic cannabis controversies and clinical trial design issues. Front Pharmacol. 2016;7:309.

17. Welty TE, Luebke A, Gidal BE. Cannabidiol: promise and pitfalls. Epilepsy Curr. 2014;14:250-252.

18. Cabral GA. Marijuana and cannabinoids. Effects on infections, immunity, and AIDS. J Cannabis Ther. 2001;1:61-85.

19. Friedman $\mathrm{H}$, Newton $\mathrm{C}$, Klein TW. Microbial infections, immunomodulation, and drugs of abuse. Clin Microbiol Rev. 2003;16:209-219.

20. Berdyshev EV. Cannabinoid receptors and the regulation of immune response. Chem Phys Lipids. 2000;108:169-190.

21. Massi $P$, Vaccani A, Parolaro D. Cannabinoids, immune system and cytokine network. Curr Pharm Des. 2006;12:3135-3146.

22. Moretti S, Castelli M, Franchi S, et al. Delta9-tetrahydrocannaninolinduced anti-inflammatory responses in adolescent mice switch to proinflammatory in adulthood. J Leukoc Biol. 2014;96:523-534.

23. Capettini LSA, Savergnini SQ, Da Silva RF, et al. Update on the role of cannabinoid receptors after ischemic stroke. Mediators Inflamm. 2012; 2012:824093.

24. Raborn ES, Marciano-Cabral F, Buckley NE, et al. The cannabinoid delta9-tetrahydrocannabinol mediates inhibition of macrophage chemotaxis to RANTES/CCL5 through the CB2 receptor. J Neuroimmun Pharmacol. 2008;3:117-129.

25. Ashton $\mathrm{CH}$. Pharmacology and effects of cannabis: a brief review. Br J Psychiatry. 2001;178:101-106.

26. World Drug Report. 2011. United Nations Office on Drugs and Crime: Vienna, Austria, 2011.

27. Radwan MM, Elsohly MA, Slade D, et al. Biologically active cannabinoids from high-potency Cannabis sativa. J Nat Prod. 2009;72:906-911.

28. Ahmed SA, Ross SA, Slade D, et al. Minor oxygenated cannabinoids from high potency Cannabis sativa L. Phytochemistry. 2015;117:194-199.

29. Frazzetto G. Does marijuana have a future in pharmacopoeia? EMBO Rep. 2003;4:651-653.

30. Crean RD, Crane NA, Mason BJ. An evidence based review of acute and long-term effects of cannabis use on executive cognitive functions. J Addict Med. 2011;5:1-8.

31. Lorke D. A new approach to practical toxicity testing. Arch Toxicol. 1983; 54:275-287.

32. Peters W. Drug resistance in Plasmodium berghei. I. Chloroquine resistance. Exp Parasitol. 1965;17:80-89.

33. Duran $M$, Pérez E, Abanades $S$, et al. Preliminary efficacy and safety of an oromucosal standardize cannabis extract in chemotherapy-induced nausea and vomiting. Br J Clin Pharmacol. 2010;70:656-663.

34. Russo EB, Marcu J. Cannabis pharmacology: the usual suspects and a few promising leads. Adv Pharmacol. 2017;80:67-134.

35. Ames FA. A clinical and metabolic study of acute intoxication with Cannabis sativa and its role in the model psychoses. J Ment Sci. 1958;104:972-999. 
36. Scotter EL, Abood ME, Glass M. The endocannabinoid system as a target for the treatment of neurodegenerative disease. Br J Pharmacol. 2010; 160:480-498.

37. Eisenstein TK, Meissler JJ. Effects of cannabinoids on T-cell function and resistance to infection. J Neuroimmun Pharmacol. 2015;10:204-216.

38. Katchan V, David P, Shoenfeld Y. Cannabinoids and autoimmune diseases: a systematic review. Autoimmun Rev. 2016;15:513-528.

39. Campos AC, Brant F, Miranda AS, et al. Cannabidiol increases survival and promote rescue of cognitive function in a murine model of cerebral malaria. Neuroscience. 2015;289:166-180.

40. Medzhitov R, Schneider DS, Soares MP. Disease tolerance as a defense strategy. Science. 2012;335:936-941.

41. Angulo I, Fresno M. Cytokines in the pathogenesis of and protection against malaria. Clin Diagn Lab Immunol. 2002;9:1145-1152.

42. Artavanis-Tsakonas $\mathrm{K}$, Tongren JE, Riley EM. The war between the malaria parasite and the immune system: immunity, immunoregulation and immunopathology. Clin Exp Immunol. 2003;133:145-152.

43. Hall W, Degenhardt L. Adverse health effects of non-medical cannabis use. Lancet. 2009;374:1383-1391.

44. Laishram DD, Sutton PL, Nanda N, et al. The complexities of malaria disease manifestations with a focus on asymptomatic malaria. Malar J. 2012;11:29.

45. Gopinath S, Lichtman JS, Bouley DM, et al. Role of disease-associated tolerance in infectious superspreaders. Proc Natl Acad Sci U S A. 2014;111:15780-15785.
46. Campbell WE, Gammon DW, Smith $P$, et al. Composition and antimalarial activity of in vitro of the essential oil of Tetradenia riparia. Planta Medica. 1997;63:270-272.

Cite this article as: Akinola O, Ogbeche EO, Olumoh-Abdul HA, Alli-Oluwafuyi AO, Oyewole AL, Amin A, AbdulMajeed Wl, Olajide OJ, Nafiu AB, Njan AA, Olorundare OE, Gbotosho GO (2018) Oral ingestion of Cannabis sativa: risks, benefits, and effects on malaria-infected hosts, Cannabis and Cannabinoid Research 3:1, 219-227, DOI: 10.1089/ can.2018.0043.

\section{Abbreviations Used}

$\mathrm{CFV}=$ cresyl fast violet

$\mathrm{H} \& \mathrm{E}=$ hematoxylin and eosin

NDLEA $=$ National Drug Law Enforcement Agency

$\mathrm{PFC}=$ prefrontal cortex

\section{Publish in Cannabis and Cannabinoid Research}

Cannabis and

Cannabinoid

Research
- Immediate, unrestricted online access

- Rigorous peer review

- Compliance with open access mandates

- Authors retain copyright

- Highly indexed

- Targeted email marketing

liebertpub.com/can 